\title{
BUILDING OF POWER BACKUP SYSTEM WITH PARALLEL CONNECTION TO GRID
}

\author{
Rodions Saltanovs $^{1,2}$, Aivars Rubenis ${ }^{1,3}$ \\ ${ }^{1}$ SIA “TransfoElectric”, Latvia; ${ }^{2}$ Riga Technical University, Latvia; ${ }^{3}$ Latvia University of Life \\ Sciences and Technologies, Latvia \\ r.saltanovs@transfoelectric.com, aivars.rubenis@transfoelectric.com
}

\begin{abstract}
The paper discusses the principles of building uninterruptible power supply systems for backup energy provision for remote or stand-alone energy consumers with inclusion of renewable energy sources. There is a problem for many energy system operators to provide energy connections for outlying clients, like stand-alone farms. These connections cost too much and are a burden both to the energy system operators and clients. In order to decrease these grid connection costs by introducing additional renewable resources a new type of inexpensive energy flow management and energy storage system was developed, which would optimize grid transmission energy flows for consumers, who occasionally need high power levels for short time periods. This paper describes the characteristics of inverters with direct connection to the power grid, the criteria for the efficiency of the uninterruptible power supply power system. Based on the analysis of the features of the operation of the main types of uninterruptible power supply units, the principle of building an efficient uninterruptible power supply system based on a converter with direct connection to the electrical grid controlled by a digital system with tracking of the maximum power consumption point is justified. The technical characteristics of the power system were determined and evaluated, the conceptual diagrams of the control device of the energy system of uninterrupted power supply and the algorithms of its operation were developed. The proposed solutions are tested experimentally by the technical implementation of the developed schemes.
\end{abstract}

Keywords: renewable energy, uninterruptible power supply.

\section{Introduction}

In order to achieve the environmental targets agreed in the Paris Convention to decrease $\mathrm{CO}_{2}$ production in energy production, local renewable energy generation is now one of the emerging technologies. Lots of research have been done in this area, including incorporating energy storage systems[1], including in Latvia[2].

In Latvia the benefits of microgeneration are now also closely examined by energy transmission system operators (TSOs). Because of low population density, TSOs have to build and maintain long distance energy lines for few users. Most of these cases are rural farms with uneven power use. This leads to high costs and transmission network charges, as TSOs have to reserve a certain power capacity for these consumers, just in case they might want the energy.

Therefore, TransfoElectric started a new project to develop an inexpensive microgrid technology for optimization of grid transmission lines for outlying small energy consumers, who occasionally need high power levels for short time periods, by incorporating renewable energy sources, like PV energy and wind energy.

The PV energy usage was analyzed in detail in [3], and this article will focus more on technical aspects of integrating wind energy creating a backup energy reserve system.

At the moment, systems working autonomously are well developed, saving energy in the battery and using it as needed. However, in the case of a centralized energy supply system, a number of questions arise about the sharing of these systems. In particular, synchronization of frequencies of alternating current and control of energy flows is necessary for joint operation of the systems. This article will discuss the issue of synchronization of the local power generation system with the power grid using renewable resources.

\section{Materials and methods}

To compare the systems in terms of efficiency it is enough to calculate the energy loss in all conversion stages. Since the power system contains a grid tied inverter (GTI) that operates on an AC network, an important parameter is the current nonlinear distortion coefficient (CNDC), a quantity expressing the degree of nonlinear distortion of the output current, equal to the ratio of the root-meansquare value of all higher current harmonics to the current of the first harmonic. CNDC determines the quality of energy delivered to the grid. 
The power systems may be either DC, like PV cells, or AC, like a local microgeneration wind turbine. To implement any algorithm tracking the maximum power point (MPP), the power system must be able to change the power conversion. The smaller the discreteness step of the power conversion, the more accurately the current power can approach the ideal MPP.

The economic efficiency of the power system will be determined by both the cost of the power system itself and additional costs or savings caused by the introduction of this power system into the design of wind turbines as a whole.

For the operation of the GTI circuit it will be necessary to control the voltage and current of the power supply network, as well as the voltage at the input of the GTI. And for the operation of the BOOST converter, data on the voltage at its input and output are necessary. To implement the search and hold algorithm for MPP, it will be necessary to obtain data on the generator speed, voltage and current values at its output. To collect these data current and voltage sensors were introduced into the power plant circuit.

The block diagram of the proposed energy system of the renewable power source (in this case wind turbine) is shown in Fig.1.

Voltage from the generator $\mathrm{G}$ is served on a three-phase rectifier assembled according to the Larionov scheme. Next, the voltage goes to the DC-DC converter, and after it on the ITT.

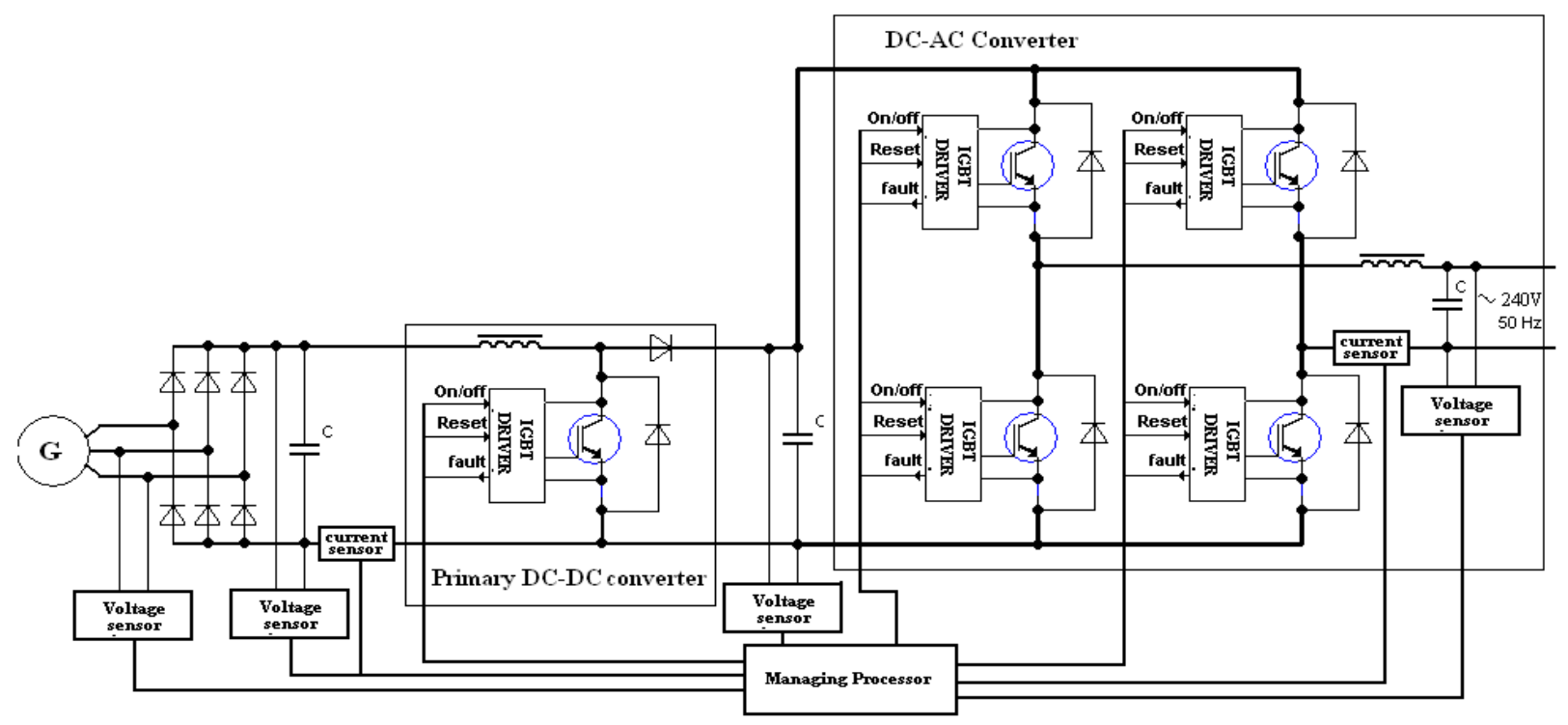

Fig. 1. Structural diagram of power system of energy generator

The circuit of the slave inverter is shown in Fig.2. It is based on the $\mathrm{H}$ bridge on the IGBT transistors. Due to the voltage on DC, if the side is higher than the peak value of the sinusoid of the power grid, then, when two transistors are opened diagonally across the bridge, the current flows from the capacitor to the power grid. In this case, thanks to the inductor connected in series, the current in the circuit will increase linearly. The inductor L 1 is selected in such a way that the current in it increases at a speed of no more than one ampere per microsecond with a maximum voltage difference between the network and capacitors equal to 320 volts. This ensures the operation of the inductor in the continuous current mode a significant part of the half-cycle of the electrical network. This mode is characterized by the fact that a direct current component flows through the choke, while the peak value current through the choke and the key element is greatly reduced compared with the regime of discontinuous current of the choke at the same converted power. The operation of the grid-tied inverter in the continuous current choke mode is shown in Fig. 2.

\section{Development of software for operation of the power system}

As shown above, the proposed wind power system consists of an intermediate converter unit and a network unit operating directly with the power grid. Both of these units must operate synchronously, ensuring the transfer of power from the generator of the wind turbines to the grid. Since the current 
and voltage received from the wind turbine generator vary within wide limits, for synchronous operation of both blocks it is expedient to use their product, ie. power. Thus, each function of the system can be distinguished into a separate program block, which increases the flexibility of the system and the convenience of the writing software. The block diagram of the software is presented in Fig. 3.

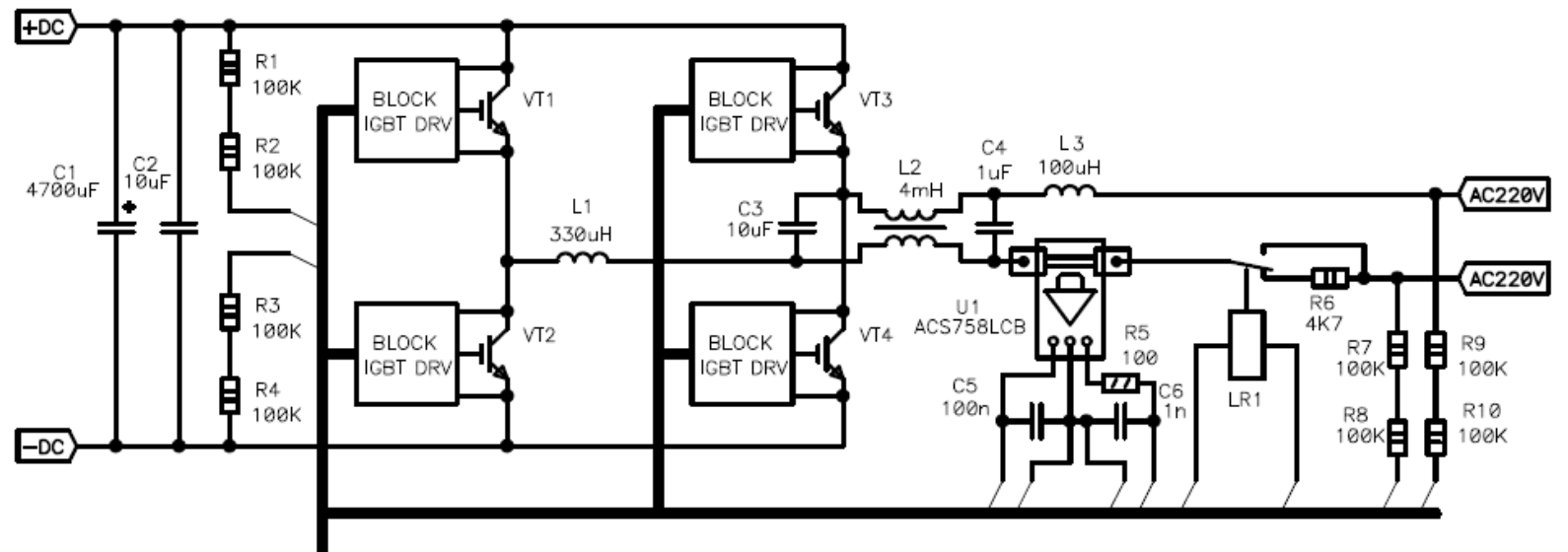

To control block.

Fig. 2. Structural diagram of grid-tied inverter

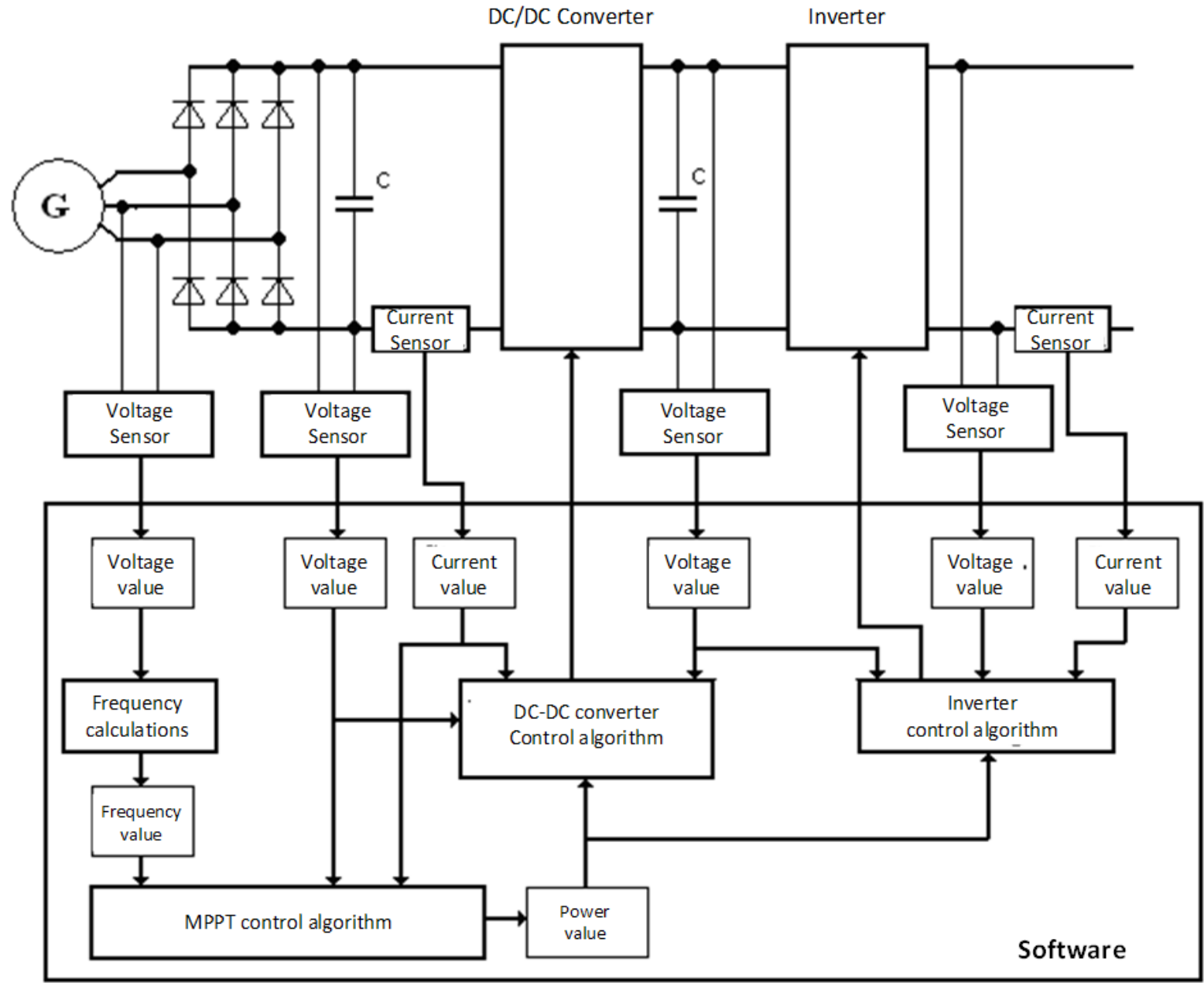

Fig. 3. Software block diagram

In the general program the following structural blocks can be distinguished:

- block processing algorithm data from sensors; 
- control block of the network block includes a program to control the $\mathrm{H}$ bridge, and the PLL;

- block Algorithm Control Algorithm Converter;

- block control algorithm for the entire wind turbine with the implementation of tracking MPP.

Data transfer from sensors to program blocks, as well as data between the blocks, is conveniently implemented using intermediate registers in the memory of the controlling controller. The execution of each program block is tied to a specific event, which can be generated by both internal and external sources. Figure 4 shows the execution the algorithm for the main blocks of the program.
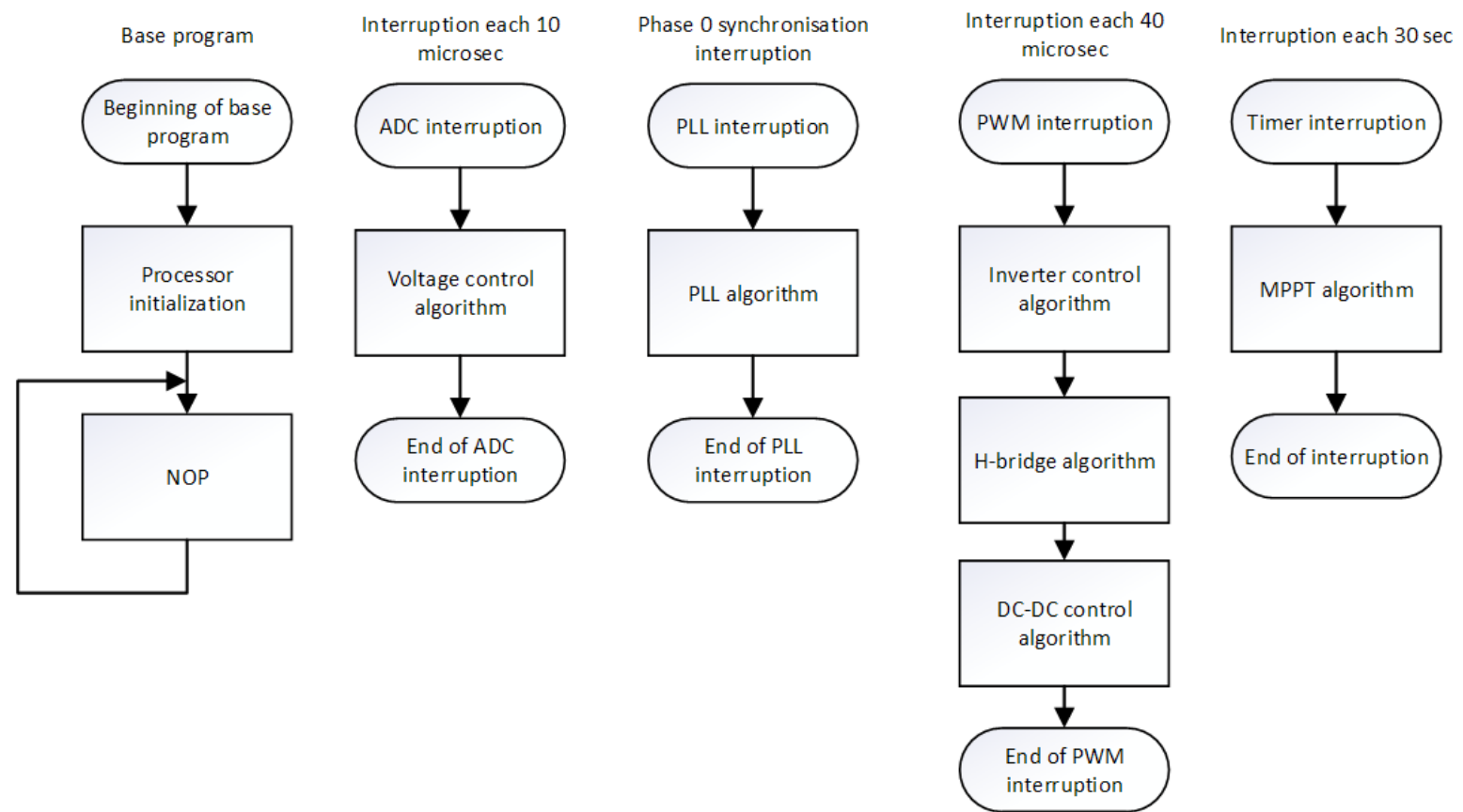

Fig. 4. Algorithm of execution of main program blocks

The highest priority is given to processing of the ADC data, since the operation of all the other program blocks requires instantaneous values of current and voltage. The algorithm for processing data from the ADC is a digital filtering, bringing the values to a common form and storing them in separate memory cells. The second priority of execution is the interruption of the PWM modulator, which is called after each timed operation of the converter to calculate and load the PWM fill values for the next cycle. The PLL algorithm and the MPP lookup have the lowest priority and are performed in the background.

\section{Results and discussion}

In the course of testing the concept of implementing an integrated power supply system, a prototype of an inverter driven by a network connected in parallel with the load to the power grid, with a capacity of $10 \mathrm{KW}$, was assembled. The main power unit of the inverter is shown in Fig. 5.
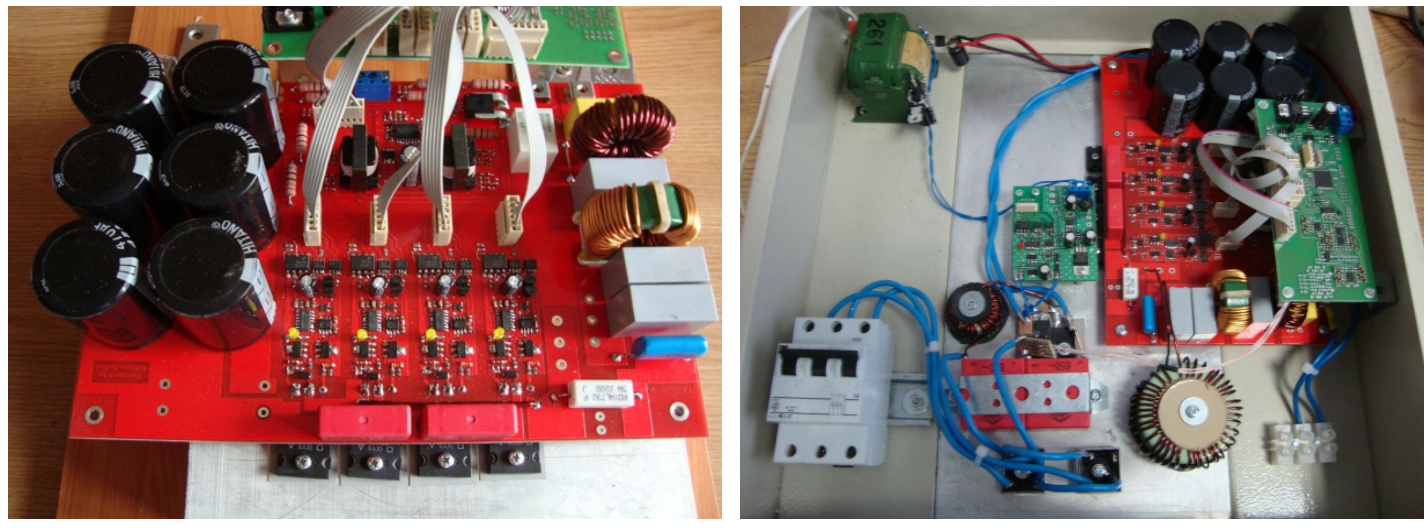

Fig. 5. Algorithm of execution of main program blocks 
The main task of the system was to provide local consumers from an auxiliary source of energy, without the return of energy to the grid. To accomplish this task the current sensors monitoring the current consumption from the power grid have been introduced into the system. Due to the superposition of currents, the controlling controller selected such a mode of the system that the load was powered from a local source, and the lack of energy was consumed from the mains. The oscillogram of the current of the inverter, displaying one half-period, is shown in Fig. 6.

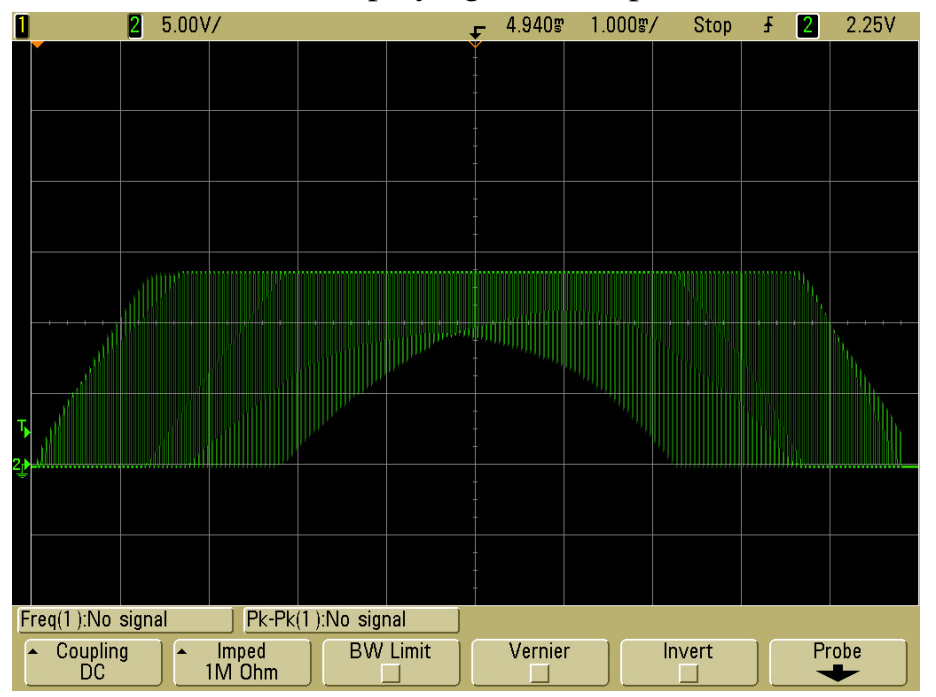

Fig. 6. Oscillogram of grid half-cycle generated with equipment

During the tests the load was used in the form of a heating element with a capacity of $5 \mathrm{~kW}$. The power of the inverter was enough to provide power to the load by $60 \%$, the rest of the power was obtained from the network. The inverter efficiency was $86 \%$.

\section{Conclusions}

In the course of this work the concept of auxiliary local power supply was developed in conjunction with the main power grid. The main difference of the developed solution is the lack of energy return in the external power grid. All energy generated by the local power source is consumed exclusively by the local consumer. The performance of this solution was tested in real-life conditions. The installation showed good efficiency and the ability to work tied in with the grid.

\section{Acknowledgements}

This paper has been published within the research project "Research on energy flow control and management algorithms and development of prototype for independent energy system based on renewable energy resources" carried out within a grant program by the European Regional Development Fund for general industrial research and for projects dealing with new product and technology developments, Project number: 1.2.1.1./16/A/008.

\section{References}

[1] Yang Y., Bremner S., Menictas C., Kay M. "Battery energy storage system size determination in renewable energy systems: A review," Renew. Sustain. Energy Rev., vol. 91, no. January, 2018, pp. 109-125.

[2] Zoss T., Rosa M., Romagnoli F., Gusca J., Blumberga D. "Modelling of electricity accumulation from irregular renewable energy resources," 27th Int. Conf. Effic. Cost, Optim. Simul. Environ. Impact Energy Syst. ECOS 2014, 2014.

[3] Rubenis A., Adrian L. R. "Determining energy storage amount for development of novel microgrid energy flow optimization system with photovoltaic energy generation," in Energy Procedia, 2018. 